\title{
Uso de Procedimentos Operacionais Padrão (POPs) comportamentais na realização de atividades profissionais
}

\author{
Geovane Testa Corrêa ${ }^{1, *}$, Aline Battisti Archer ${ }^{1}$, \\ Gustavo Klauberg Pereira ${ }^{2}$, Juliane Viecili ${ }^{3}$
}

${ }^{1}$ ÂMBIA - Comportamento Humano nas Organizaçoes, Brasil

${ }^{2}$ Polícia Militar de Santa Catarina (PMSC), Brasil;

${ }^{3}$ Universidade do Sul de Santa Catarina (Unisul), Brasil

Submissão: 12/03/2019

Primeira decisão editorial: 03/10/2019

Versão final: 03/10/2019

Aceite: 03/10/2019

\section{Resumo}

O objetivo desta pesquisa foi caracterizar resultados produzidos pelo uso de procedimentos operacionais padrão (POPs), escritos com base na noção de comportamento (POP-Cs), na realização de atividades profissionais. Foram desenvolvidos dois estudos complementares, no âmbito de atividades profissionais que ocorrem em softwares de gestão, em uma organização privada brasileira. O Estudo 1 refere-se à avaliação da eficácia de POP-Cs em relação a POPs previamente existentes na organização, no qual foram realizados testes com cinco profissionais. O Estudo 2 refere-se à identificação de benefícios e limitações percebidos por usuários de POP-Cs, no qual foram realizadas entrevistas com três profissionais. O uso de POP-Cs viabilizou, em 100\% das vezes, a realização das atividades pelos profissionais, o que não ocorreu com os POPs previamente existentes na organização, gerou sentimentos positivos nos profissionais que nunca haviam realizado a atividade descrita e auxiliou na elaboração do treinamento de novos profissionais.

Palavras-chave: modelagem do processo, capacitação profissional, análise do comportamento.

\section{Results of the use of behavioral SOPs in the perfor- mance of professional activities}

\begin{abstract}
This research aimed to identify results produced by the use of Standard Operating Procedures (SOPs), written under the control of behavioral concepts (B-SOPs), in the accomplishment of professional activities. Two studies were carried out in a Brazilian organization and involved the use of B-SOPs in professional activities that occur in the context of business management software. Study 1 refers to the evaluation of B-SOPs efficacy in relation to previously existent SOPs in the organization. Study 2 refers to the identification of benefits and limitations noticed by three professionals that used de B-SOPs in their activities. The use of B-SOPs made possible, in $100 \%$ of the times, the accomplishment of the professional activities, what didn't happen with the previously existent SOPs in the organization. Besides that, the use of B-SOPs generated positive feelings among the professionals that performed the activities, and it aided the instructional design's elaboration for new employees.
\end{abstract}

Keywords: workflow, professional training, behavior analysis.
Resultados del uso de POEs conductuales en la realización de actividades profesionales

\begin{abstract}
Resumen
Esta investigación objetivó caracterizar los resultados producidos por el uso de Procedimientos Operacionales Estandarizados (POEs), escritos con base en la noción de conducta (POE-Cs), en la realización de actividades profesionales. Dos estudios complementares fueron desarrollados en una organización brasileña, en el ámbito de actividades profesionales que ocurren en softwares de gestión. El Estudio 1 se refiere a la evaluación de la eficacia de POE-Cs en relación con POEs antes existentes en la organización. El Estudio 2 se refiere a la identificación de beneficios y limitaciones notadas por usuarios de POE-Cs. El uso de POE-Cs hizo posible, en 100\% de las pruebas, el logro de las actividades por los profesionales, lo que no ocurrió con los POEs antes existentes en la organización, generó sentimientos positivos en los profesionales que nunca habían hizo la actividad descrita y ayudó en la elaboración del entrenamiento de nuevos profesionales.
\end{abstract}

Palabras-clave: flujo de trabajo, capacitación profesional, análisis de la conducta.
* Informações sobre o autor principal:

Endereço de correspondência: Rua Santa Luzia, 100, sala 306, Trindade, Florianópolis (SC), Brasil, CEP 88.036-540.

E-mail: geovane@ambiacomportamento.com.br 
Nas organizações, é um desafio propor ações profissionais que promovam benefícios às pessoas e, com certa frequência, profissionais deixam de produzir benefícios àqueles que necessitam de sua intervenção. Por exemplo, nos Estados Unidos, pesquisadores têm mostrado que, anualmente, pelo menos 75 mil mortes nas cirurgias decorrem de erros em procedimentos médicos (Gawande, 2009). No Brasil, é relevante destacar os casos nos quais profissionais da área da saúde levaram pacientes a óbito, quando injetaram substâncias indesejadas na corrente sanguínea desses pacientes. Em um caso, foi injetado café (Folha de São Paulo, 2012) e, no outro, um composto químico utilizado em processos industriais (Veja, 2013). Segundo os autores das reportagens, os profissionais estavam atuando há menos de 15 dias e sem supervisão técnica quando os problemas ocorreram. Erros como os descritos poderiam ser prevenidos, se os profissionais tivessem clareza acerca das atividades que deveriam realizar no exercício de seu cargo. O uso do procedimento operacional padrão (POP) é um dos meios para tornar conhecidas, estáveis e acessíveis tais atividades, bem como para viabilizar que essas sejam gerenciadas de modo efetivo nas organizações.

O POP, também denominado de procedimento, instrução de trabalho (Araújo, 2010) ou protocolo (Maranhão \& Macieira, 2010), é um documento em que consta a descrição de como uma atividade deve ser realizada e de informações relacionadas a essa atividade (Araújo, 2010; Campos, 2014; Harris, 2014; Reeuwijk \& Houba, 1998). Seu objetivo é viabilizar que uma atividade seja realizada adequadamente e sempre da mesma forma por diferentes pessoas, de modo a garantir, com segurança e eficiência, o atendimento das necessidades dos clientes (Campos, 2014; Reeuwijk \& Houba, 1998).

No âmbito da gestão de processos, o POP contém a descrição da menor unidade de um processo: a tarefa (Association of Business Process Management Professionals [ABPMP], 2013), cuja contextualização nesse âmbito está representada na Figura 1. No nível mais abrangente, é possível observar o processo, que é constituído por diferentes atividades interdependentes, ordenadas no tempo e no espaço, e que tem entradas e saídas definidas. As atividades, por sua vez, são constituídas por tarefas encadeadas de modo lógico, que geram um resultado final. E cada tarefa se refere à ação que o profissional realiza em uma atividade (ABPMP, 2013). Portanto, a tarefa é o elemento observável da atuação de um profissional e o POP é o documento que contém a descrição do grau mais refinado de decomposição de um processo.

Como decorrência desse grau de descrição, o uso do POP, quando bem escrito, aumenta as chances de produzir benefícios, direta ou indiretamente, para os envolvidos na atividade padronizada. Entre as consequências, viabiliza que: (1) o profissional execute a atividade sem depender de ordens frequentes de seu gestor, tenha mais segurança ao realizar uma atividade e maiores chances de atender às expectativas da organização em relação ao seu desempenho; (2) o gestor treine novos funcionários de modo que não restrinja o treinamento apenas a instruções genéricas e orais, deixe de procurar solução para o mesmo problema repetidas vezes, deixe de interferir de modo frequente na execução da atividade do profissional pelo qual é responsável e planeje sua rotina de trabalho e dos demais integrantes de sua equipe; (3) o cliente receba um produto com as mesmas características e com o mesmo prazo de entrega ao longo do tempo, aumentando as chances de fidelização; e (4) a organização tenha assegurado seu domínio tecnológico, de modo a não depender do conhecimento de pessoas específicas (Campos, 2014; Silva, Duarte, \& Oliveira, 2004).

$\mathrm{Na}$ literatura revisada, existem manuais com indicações de como redigir sentenças que expressem tarefas, com o objetivo de o POP atender à função a qual se destina. Segundo esses manuais, as tarefas devem ser escritas no formato passo a passo (Empresa Brasileira de Serviços Hospitalares, 2014; United States Environmental Protection Agency [US EPA], 2001) e de forma clara e concisa (Peixoto, Marques, Silva, \& Ferraz, 2015; Rateke, 2014; US EPA, 2001). Além disso, o elaborador do POP deve evitar o uso de termos ou expressões que possam gerar diferentes interpretações pelo leitor (Peixoto et al., 2015; US EPA, 2001) e prezar pela compreensão do usuário em relação ao que está escrito (Peixoto et al., 2015). Embora essas sejam instruções que possam orientar em algum grau a escrita de um POP, elas ainda são genéricas. Afinal, o que caracteriza uma escrita clara e concisa? Como escrever uma sentença que expresse, de modo inequívoco, o que o profissional deve fazer? Diante das características dessas instruções e da ausência de definição precisa dos aspectos centrais que constituem a redação de sentenças que expressam uma tarefa, é possível afirmar que não há conhecimento suficientemente consolidado acerca do que caracteriza a escrita de uma tarefa, que garanta que seja realizada da mesma forma por diferentes pessoas, sem produzir consequências indesejáveis à organização.

No âmbito da análise do comportamento, subárea de conhecimento da psicologia, a noção de comportamento parece constituir uma contribuição relevante para a elaboração de sentenças que expressam uma tarefa. Se tarefa é a ação que o profissional realiza (ABPMP, 2013) e comportamento é a relação entre aspectos daquilo que a pessoa faz (ação), certas características da situação

\section{PROCESSO}

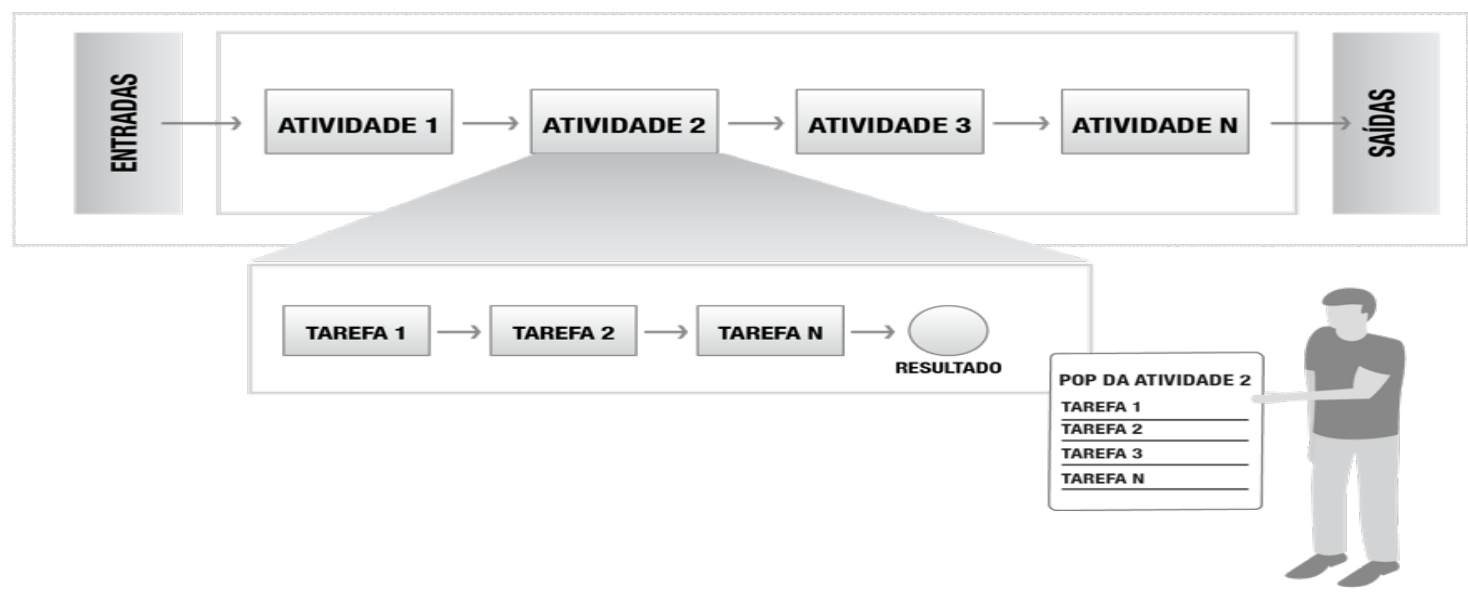

Figura 1. Representação das relações de abrangência entre processo, atividade e tarefa no âmbito da gestão de processos, e das tarefas escritas em um procedimento operacional padrão (POP). É no nível de abrangência da tarefa que ocorre aquilo que é observável na atuação do profissional, e as tarefas escritas são as orientações para o profissional realizar efetivamente a atividade no ambiente de trabalho. 
antecedente a essa ação e certas características da situação subsequente a essa ação (Botomé, 2013), então, é possível afirmar que a noção de comportamento traz mais visibilidade em relação ao que caracteriza uma tarefa. Consequentemente, quando um elaborador de um POP utiliza a noção de comportamento para descrever uma tarefa, ele evidencia, além da própria ação de interesse, as variáveis relevantes relacionadas a essa ação, o que aumenta as chances de o registro da tarefa corresponder exatamente àquilo que ocorre na realização de uma atividade e de ser realizável por diferentes pessoas. Nesse sentido, uma sentença que expressa uma tarefa, considerando a perspectiva comportamental, além de ser escrita de modo claro ao seu usuário, deve conter componentes do comportamento.

A noção de comportamento amplia a compreensão do que caracteriza uma tarefa e, portanto, do que deve ser registrado em relação a ela. Essa forma de caracterização de uma tarefa, por sua vez, provavelmente torna o "POP comportamental" uma ferramenta útil aos profissionais que o utilizam. No entanto, na literatura revisada, ainda não são conhecidos os benefícios e as limitações de uso, nas organizações, de um POP escrito explicitamente com base na noção de comportamento (POP-Cs). Diante disso, o objetivo desta pesquisa foi caracterizar resultados produzidos pelo uso de POP-Cs na realização de atividades profissionais. De modo a alcançar esse objetivo, foram desenvolvidos dois estudos complementares: o Estudo 1, referente à avaliação da eficácia de POP-Cs em relação a POPs previamente existentes na organização onde a investigação ocorreu; e o Estudo 2, referente à identificação de benefícios e limitações percebidos por usuários de POP-Cs.

\section{Método}

Os estudos foram realizados em uma organização privada, distribuidora de alimentos no Sul do Brasil, fundada em 1987 e composta por 106 profissionais. Os estudos fazem parte de um programa de pesquisa relacionado ao desenvolvimento de técnicas de desenho de processos comportamentais em organizações, iniciado em 2012. Esta pesquisa empírica refere-se a um dos projetos do programa, que tem como objetivo o desenvolvimento de uma técnica para a descrição de atividades profissionais que envolvem a utilização de softwares de gestão como meio para realizar diferentes trabalhos desenvolvidos em organizações.

\section{Estudo 1. Avaliação da Eficácia de POP-Cs em Relação a POPs Previamente Existentes na Organização}

Participantes. Participaram deste estudo quatro profissionais da organização e um candidato a jovem aprendiz. De forma a garantir uma variabilidade significativa de repertório comportamental, o grupo de participantes foi formado por pessoas com faixa etária entre 14 e 75 anos de idade e diferentes graus de escolaridade, conforme pode ser observado na Tabela 1. Os participantes foram selecionados a partir de quatro critérios principais: (a) não poderia ter realizado previamente a atividade profissional descrita; (b) deveria ter completado, pelo menos, os anos iniciais do ensino fundamental; (c) ter conhecimento básico no uso de computador; e (d) ter conhecimento básico no uso de softwares de quaisquer tipos de sistemas operacionais usualmente existentes em computadores ou smartphones.

Instrumentos. Para avaliar a eficácia de POP-Cs em relação a POPs previamente existentes na organização, foram utilizados dois instrumentos: POPs previamente existentes na organização e POPs escritos com base na noção de comportamento (POP-Cs).

Os POPs previamente existentes na organização foram escritos, pelos profissionais da própria organização, com as seguintes características: (a) no formato passo a passo, de acordo com a ordem com que o profissional deveria realizar a atividade; (b) com objetividade, a fim de conter somente o que fosse relevante; e (c) em linguagem compreensível para os demais colegas.

Os POP-Cs foram desenvolvidos para atividades profissionais que ocorrem em softwares de gestão (em geral conhecidos como Enterprise Resource Planning [ERP]) e foram caracterizados por: (a) cada sentença ser escrita com base na noção de comportamento; (b) cada sentença ser escrita para um profissional que nunca executou a atividade profissional descrita; (c) cada sentença ser escrita com a função de ser realizável por um profissional com os anos iniciais do ensino fundamental completos, conhecimento básico no uso de computador e conhecimento básico no uso de softwares de sistemas operacionais usualmente existentes em computadores ou smartphones; e (d) cada linha conter somente uma sentença.

Tabela 1

Características dos participantes do Estudo 1

\begin{tabular}{ccc}
\hline Participante & Idade & Escolaridade \\
\hline P1 & 27 & Mestrado \\
P2 & 29 & Superior \\
P3 & 66 & Médio \\
P4 & 14 & Fundamental \\
P5 & 75 & incompleto \\
\hline
\end{tabular}

A sentença comportamental foi escrita conforme a seguinte ordem: (1) no primeiro termo da sentença estava escrito o local relevante onde a ação do profissional deveria ocorrer; (2) no segundo termo da sentença estava escrita a ação relevante expressa por um verbo no infinitivo; e (3) no terceiro termo da sentença estava escrito o complemento relevante em relação à ação, podendo ser qualquer objeto do ambiente utilizado na ação do profissional e/ou uma característica relevante dessa ação. Sendo a palavra "objeto" definida como "tudo que é perceptível por qualquer dos sentidos" (Ferreira, 2004). Exemplos: (a) "No teclado, apertar a tecla 4", em que "No teclado" (local), "apertar" (ação) "a tecla 4" (o objeto do ambiente utilizado na ação); (b) "No teclado, apertar duas vezes a tecla 4", em que "No teclado" (local), "apertar" (ação) "duas vezes" (característica da ação) "a tecla 4" (o objeto do ambiente utilizado na ação).

Por fim, as sentenças comportamentais foram dispostas no texto, de modo que um profissional produzisse o resultado final da atividade após completar a realização de todas as sentenças comportamentais.

Procedimentos de coleta de dados e cuidados éticos. Foram selecionadas quatro atividades profissionais a serem realizadas pelos participantes por meio dos POPs previamente existentes na organização e dos POP-Cs: três atividades do setor financeiro e uma do setor de transporte. Tal seleção levou em conta três aspectos principais: (a) a atividade já deveria estar descrita em um POP, conforme as características apresentadas na seção "POPs previamente existentes na organização"; (b) o tempo para realização das quatro atividades deveria levar em torno de uma hora de trabalho; e (c) as atividades deveriam ser realizadas em interação com o software de gestão.

Os participantes realizaram as atividades, descritas nos POPs previamente existentes na organização e nos POP-Cs, em estações de trabalho usualmente encontradas nas organizações: com cadeira, mesa e computador para acessar os softwares específicos relacionados aos dois tipos de POPs em avaliação. Os procedimentos foram realizados individualmente, em uma sala privativa na qual não poderiam ser interrompidos $\mathrm{e}$ onde estavam presentes somente o pesquisador e o participante.

Cada atividade tinha dois POPs escritos de formas diferen- 
tes: o previamente existente na organização e o POP-C. Antes da realização das atividades, os participantes receberam uma explicação acerca do contexto do estudo, do trabalho a ser realizado e de como deveriam ser lidos os dois tipos de procedimentos. As quatro atividades foram realizadas em sequência, seguindo uma ordem de execução pelo participante: primeiro foi entregue o POP previamente escrito na organização referente à primeira atividade a ser realizada e, imediatamente após a realização desta, foi entregue o POP-C referente à mesma atividade. $\mathrm{O}$ mesmo procedimento foi realizado em relação às demais.

Para definir a ordem de execução dos POPs, foi considerada a seguinte premissa: o procedimento escrito de modo mais completo tende a aumentar as chances de ser produzido o resultado da atividade. Considerando isso, o POP previamente escrito na organização foi o primeiro aplicado, pois continha menor quantidade de sentenças que o POP-C e, portanto, era o menos completo.

Durante a realização da atividade, o pesquisador registrou em uma planilha auxiliar um símbolo que indicava se o participante tinha realizado com sucesso ou não a tarefa. Além disso, dúvidas e dificuldades dos participantes foram assinaladas. O pesquisador não interferiu na realização das atividades descritas nos dois tipos de POPs.

A execução do estudo 1 levou em conta procedimentos éticos, havendo consentimento por parte da direção da organização e dos profissionais que participaram das diferentes etapas do estudo.

Procedimentos de análise de dados. Os dados registrados na planilha auxiliar foram transferidos para planilhas eletrônicas nas quais constavam as sentenças dos dois tipos de POPs avaliados. Após a transferência, os resultados de cada um dos participantes foram consolidados por tipo de POP e de atividade profissional.

\section{Estudo 2. Identificação de Benefícios e Limitações Percebi- dos por Usuários de POP-Cs}

Participantes. Três participantes foram entrevistados a fim de obter informações mais precisas sobre benefícios e limitações no uso de POP-Cs. Esses participantes eram dois estagiários recém contratados na organização, que nunca tinham tido contato com POP-Cs, e o treinador deles. Um participante era do sexo feminino, tinha 16 anos, estudante da $1^{\mathrm{a}}$ série do ensino médio e contratada como jovem aprendiz, denominada P6; outro era do sexo masculino, tinha 26 anos, estudante de graduação em administração e contratado como auxiliar de controladoria trainee, denominado P7; e o terceiro participante era do sexo masculino, tinha 28 anos, ensino médio completo, dois anos de vínculo com a empresa e contratado como auxiliar de controladoria, denominado treinador (T). Todos os profissionais atuavam no setor financeiro da organização e utilizavam softwares de gestão para realizar suas atividades.

Os participantes foram selecionados por decorrência de uma situação de trabalho na qual foi utilizada um POP-C. Nessa situação, o treinador precisava ensinar a atividade profissional "cadastrar cliente pessoa jurídica" tanto para P6, quanto para P7, pois iria entrar de férias e delegar essa atividade futuramente a P7. A aprendiz P6 seria auxiliar de P7 após a atividade ser delegada a esse último. De forma a auxiliar no planejamento e na realização do treinamento dos dois participantes, foi elaborado um POP-C da atividade profissional mencionada.

Instrumentos. De forma a identificar benefícios e limitações percebidos por usuários de POP-Cs, foram realizadas entrevistas com os participantes por meio de um roteiro semiestruturado caracterizado predominantemente por perguntas abertas.

Procedimentos de coleta de dados e cuidados éticos. A coleta dos dados foi realizada por dois entrevistadores em sala privativa na própria empresa, livre de ruídos e interrupções, e feita individualmente. As informações foram registradas de forma manuscrita durante a realização da entrevista e, posteriormente, os registros individuais dos entrevistadores foram comparadas e complementadas em um único registro.

A execução do estudo 2 levou em conta procedimentos éticos, havendo consentimento por parte da direção da organização e dos profissionais que participaram das diferentes etapas do estudo.

Procedimentos de análise de dados. Para organização e tratamento dos dados das entrevistas foram realizados dois procedimentos: análise de conteúdo e elaboração de categorias. As categorias foram elaboradas a priori, a partir das questões apresentadas no roteiro de entrevista, totalizando quatro. Em seguida, foram elaboradas subcategorias a partir das falas dos participantes, totalizando 19, distribuídas de forma não equitativa nas categorias. Os trechos dos relatos selecionados foram decompostos a partir de características do POP-C ou de aspectos que facilitaram ou dificultaram seu uso em relação à atividade realizada.

\section{Resultados}

\section{Estudo 1. Avaliação da Eficácia de POP-Cs em Relação a POPs Previamente Existentes na Organização}

É relevante destacar resultados do estudo referentes a três variáveis relacionadas à eficácia dos POP-Cs em relação aos POPs previamente existentes na organização: (a) a quantidade total de sentenças por tipo de POP; (b) a proporção média com que os profissionais conseguiram realizar a atividade por meio de cada tipo de POP; e (c) a proporção dos participantes que produziu o resultado final da atividade por meio de cada tipo de POP. Os dados estão apresentados na Tabela 2, distribuídos por atividade profissional e, na atividade, por tipo de POP.

Com relação à quantidade total de sentenças, é possível observar que foram escritas 32 sentenças no conjunto de POPs previamente existentes na organização, e 71 sentenças no conjunto de POPs escritos com base na noção de comportamento; portanto, foram escritas 39 sentenças a mais nos POP-Cs que nos POPs previamente existentes na organização. Em outras palavras, e em comparação com os POPs previa-

Tabela 2

Distribuição dos resultados produz̨idos pelos cinco participantes do Estudo 1 por atividade profissional e por tipo de escrita de POP (previamente existente na organização e POP-C)

\begin{tabular}{|c|c|c|c|c|c|c|c|c|}
\hline \multirow[t]{2}{*}{ Variáveis medidas } & \multicolumn{2}{|c|}{$\begin{array}{c}\text { Atividade } \\
\text { profissional } 1\end{array}$} & \multicolumn{2}{|c|}{$\begin{array}{c}\text { Atividade } \\
\text { profissional } 2\end{array}$} & \multicolumn{2}{|c|}{$\begin{array}{c}\text { Atividade } \\
\text { profissional } 3\end{array}$} & \multicolumn{2}{|c|}{$\begin{array}{c}\text { Atividade } \\
\text { profissional } 4\end{array}$} \\
\hline & POP & POP-C & POP & POP-C & POP & POP-C & POP & POP-C \\
\hline Quantidade total de sentenças por procedimento & 4 & 14 & 11 & 16 & 11 & 28 & 6 & 13 \\
\hline $\begin{array}{l}\text { Proporção média de realização da atividade por meio do proce- } \\
\text { dimento }\end{array}$ & $15,0 \%$ & $100,0 \%$ & $21,8 \%$ & $100,0 \%$ & $6,7 \%$ & $100,0 \%$ & $42,9 \%$ & $100,0 \%$ \\
\hline $\begin{array}{l}\text { Proporção de participantes que produziu o resultado final da } \\
\text { atividade por meio do procedimento }\end{array}$ & $0,0 \%$ & $100,0 \%$ & $0,0 \%$ & $100,0 \%$ & $0,0 \%$ & $100,0 \%$ & $0,0 \%$ & $100,0 \%$ \\
\hline
\end{tabular}


mente existentes na organização, os POP-Cs viabilizaram um aumento médio de 121,9\% na "visibilidade" ou "clareza" do que os profissionais precisavam fazer nas atividades avaliadas.

A outra variável medida está relacionada à proporção média de realização da atividade por meio de cada tipo de POP. Essa variável leva em conta o quociente entre quantas tarefas escritas cada participante conseguiu efetivamente realizar em relação ao total de tarefas escritas que precisava realizar para completar cada atividade profissional. Em resumo, essa variável explicita quanto um profissional consegue efetivamente realizar do total das tarefas escritas em um POP, e pode ser nomeada como eficácia (Associação Brasileira de Normas Técnicas, 2005) de realização da atividade por meio do tipo de procedimento escrito. É possível observar que todos os participantes conseguiram realizar todas as tarefas escritas constituintes dos POP-Cs de cada atividade profissional. Ou seja, quando os POPs foram elaborados com base na noção de comportamento, o grau de realização de cada tarefa escrita foi de $100 \%$ para todos os participantes. Em contrapartida, quando foram usados os POPs previamente existentes na organização, os participantes conseguiram realizar somente uma parte das tarefas constituintes das quatro atividades profissionais avaliadas, em uma proporção média que variou de 6,7 a 42,9\%.

A terceira variável medida é consequência do quanto cada participante conseguiu realizar no âmbito da atividade profissional. Isto é, se o sujeito consegue realizar todas as tarefas de uma atividade, então, ele consegue produzir o resultado final da atividade. Quando os POPs foram elaborados com base na noção de comportamento, o índice de produção do resultado final de cada atividade foi de $100 \%$. Contudo, quando os POPs previamente existentes na organização foram utilizados pelos participantes, nenhum deles conseguiu produzir os resultados finais das atividades avaliadas.

Durante o uso dos POP-Cs, foram identificadas dificuldades dos participantes na interação com os softwares de gestão em virtude de suas características. Por exemplo, foram identificados casos em que houve dificuldade para achar o botão certo para clicar, pois ele estava muito distante de outros botões (caso identificado na atividade 3); botões nos quais o conteúdo escrito tinha pouco contraste em relação à cor, dificultando a leitura (identificado na atividade 2); ícones apareciam sem identificação ou com identificação em língua estrangeira (inglês), o que dificultava a leitura por parte dos participantes, uma vez que nenhum deles conseguia decodificar o que significava o ícone ou entender o que estava escrito em inglês.

\section{Estudo 2. Identificação de Benefícios e Limitações Percebi-} dos por Usuários de POP-Cs

Na Tabela 3 estão apresentadas as categorias e as subcategorias que representam as falas dos participantes acerca da utilização de POP-Cs. Na primeira coluna são apresentadas as categorias, na segunda coluna, as subcategorias, e, na terceira, os participantes, cujas falas em entrevista foram relacionadas às subcategorias. Conforme pode ser observado na primeira categoria - "Benefícios do POP-C", a "Efetividade na realização de tarefas" (duas ocorrências), a "Autonomia na realização da atividade" (três ocorrências) e a "Otimização do tempo de treinamento" (duas ocorrências) foram os benefícios do uso do POP-C mais destacados pelos participantes.

Em relação à categoria "Limitações do POP-C", a "Extensão excessiva" foi a mais frequentemente indicada pelos participantes (três ocorrências). Como sugestões de alteração do POP-C, foi indicada a necessidade de "Diminuir a extensão" (duas ocorrências). Dois participantes (P6 e T-P6) indicaram não haver necessidade de alterar o POP-C. Todos os participantes (P6, P7, T-P6, T-P7) sugeriram que o "Conteúdo" do POP-C fosse mantido.
Tabela 3

Representação, em categorias e subcategorias, da percepção dos participantes acerca do uso de POP-Cs

\begin{tabular}{|c|c|c|}
\hline Categorias & Subcategorias & Participantes \\
\hline \multirow{7}{*}{ Benefícios do POP-C } & $\begin{array}{l}\text { Efetividade na realização de } \\
\text { tarefas }\end{array}$ & P6, T-P6 \\
\hline & $\begin{array}{l}\text { Menor demanda de super- } \\
\text { visão }\end{array}$ & P7 \\
\hline & $\begin{array}{l}\text { Autonomia na realização da } \\
\text { atividade }\end{array}$ & P6, P7, T-P7 \\
\hline & $\begin{array}{l}\text { Segurança na realização da } \\
\text { atividade }\end{array}$ & P7 \\
\hline & Agilidade no treinamento & T-P6 \\
\hline & $\begin{array}{l}\text { Otimização do tempo de } \\
\text { treinamento }\end{array}$ & T-P6, T-P7 \\
\hline & $\begin{array}{l}\text { Ampliação do controle } \\
\text { de informações a serem } \\
\text { ensinadas }\end{array}$ & T-P6 \\
\hline \multirow{5}{*}{ Limitações do POP-C } & Manejo inicial & P6 \\
\hline & $\begin{array}{l}\text { Alternância entre leitura e } \\
\text { realização da atividade }\end{array}$ & P6 \\
\hline & $\begin{array}{l}\text { Mais esforço para o profis- } \\
\text { sional do que a instrução } \\
\text { oral }\end{array}$ & P7 \\
\hline & Extensão excessiva & P7, T-P6, T-P7 \\
\hline & $\begin{array}{l}\text { Execução sem compreensão } \\
\text { da função da atividade }\end{array}$ & T-P6 \\
\hline \multirow{6}{*}{$\begin{array}{l}\text { Sugestões de alteração do } \\
\text { POP-C }\end{array}$} & Nenhuma & P6, T-P6 \\
\hline & Diminuir a extensão & P7, T-P7 \\
\hline & $\begin{array}{l}\text { Complementar a descrição } \\
\text { de atividades }\end{array}$ & P6 \\
\hline & $\begin{array}{l}\text { Diminuir algumas especifi- } \\
\text { cidades }\end{array}$ & T-P7 \\
\hline & $\begin{array}{l}\text { Explicitar a função de sua } \\
\text { extensão }\end{array}$ & T-P6 \\
\hline & Inserir prints das telas & P7 \\
\hline $\begin{array}{l}\text { Sugestões de manutenção } \\
\text { do POP-C }\end{array}$ & Manter conteúdo & $\begin{array}{l}\text { P6, P7, T-P6, } \\
\text { T-P7 }\end{array}$ \\
\hline
\end{tabular}

Nota. A sigla T-P6 significa que o treinador fez um relato em relação à P6. A sigla T-P7, que o relato do treinador foi em relação ao P7.

\section{Discussão}

\section{Estudo 1. Avaliação da Eficácia de POP-Cs em Relação a POPs Previamente Existentes na Organização}

Uma primeira descoberta a ser destacada quanto ao uso de POP-Cs está relacionada à consistência na realização das tarefas constituintes de atividades profissionais que ocorrem com apoio de softwares. Neste estudo, quando os POPs foram elaborados com base na noção de comportamento, o índice de realização de cada tarefa escrita foi consistentemente de $100 \%$ para todos os participantes na única tentativa que tinham para realizá-la. Esse grau de consistência, por outro lado, não ocorreu em estudos que também avaliaram a eficácia de uso de POPs em atividades profissionais que ocorrem com apoio de softwares. Por exemplo, Gong, Li, Mattson, Åkerman e Berglund (2017), Li et al. (2018) e Menn, Ulbrich e Günther (2018) avaliaram a eficácia do uso de POPs na capacitação profissional de montagem de peças industriais com o apoio de diferentes tipos de tecnologia, como textos 3D-PDF, softwares de análise de produção industrial, vídeos, realidade aumentada e realidade virtual.

Esses pesquisadores descobriram que o índice de realização dos POPs variou de 50 a 100\%, de acordo com os tipos de apoio tecnológico e a quantidade de vezes que os participantes poderiam repetir o procedimento. Como em nenhum 
dos três estudos (Gong et al., 2017; Li et al., 2018; Menn et al., 2018) houve avaliação acerca do que constituía cada sentença dos POPs testados, um dos prováveis determinantes da inconsistência dos resultados pode ter sido justamente a forma como os pesquisadores escreveram as sentenças que orientaram a construção dos POPs apoiados por diferentes tipos de tecnologia.

Outra descoberta relevante de ser destacada no âmbito da capacitação profissional com o uso de POP-Cs é que, após os participantes realizarem todas as tarefas escritas nos POP-Cs, todos produziram os resultados finais de cada uma das quatro atividades profissionais. A relevância dessa descoberta decorre do fato de que a produção de resultados finais de atividades profissionais viabiliza a sustentabilidade de organizações. Afinal, sem produtos a serem entregues para clientes internos ou externos, as chances de sobrevivência das organizações são remotas. Por conta da importância desse tipo de decorrência, é que pesquisadores (Gong et al., 2017; Li et al., 2018; Menn et al., 2018) têm estudado o uso de diferentes tipos de POPs com apoios tecnológicos diversos, que sejam realizáveis por profissionais com repertório distintos, de forma que desse uso resultem os produtos requeridos pelos clientes.

Por fim, há uma descoberta importante relacionada ao desenvolvimento e teste de softwares, quando o POP-C é utilizado na realização de atividades profissionais que ocorrem com apoio de softwares: a forma de escrita da sentença comportamental pode auxiliar, os profissionais de desenvolvimento e teste, na detecção de elementos a serem aperfeiçoados no próprio software. A utilização da sentença comportamental facilitou a identificação de aspectos desnecessários na interface com o usuário e pontos precisos em que o software precisaria ser mais bem construído, de forma a facilitar a interação com o usuário. Com softwares mais bem construídos (i.e., adequados na interação com os usuários), aumentam as chances de os profissionais de informática produzirem mais eficaz e eficientemente aquilo que é esperado.

\section{Estudo 2. Identificação de Benefícios e Limitações Percebi- dos por Usuários de POP-Cs}

A utilização de POP-C produziu benefícios aos participantes do estudo. Em entrevista, dois participantes que estavam ingressando na empresa e desconheciam a atividade a ser realizada, bem como o sistema de software utilizado, relataram que o POP-C possibilitou que atingissem o resultado esperado nas atividades sem a necessidade de solicitar orientações do treinador ou de colegas, como é possível notar na fala de P7 “[...] quando eu tive uma dúvida, eu pude consultar o padrão em vez de ter consultado o treinador, até por uma questão de segurança [...]" e na fala de P6 "[...] ajudou para cadastro [de] clientes [...] Ixiii... sem a folha [POP-C] não teria nem começado [a cadastrar os clientes]". A segurança, a autonomia e a facilidade na realização de atividades decorrentes do uso de POPs também foram identificadas em pesquisas realizadas com objetivo de avaliar a implementação de POPs em organizações de saúde (Pereira et al., 2017; Sales et al., 2018). Dadas as descrições de sentimentos positivos associados à realização de diferentes atividades profissionais por meio de procedimentos escritos, é possível afirmar que tanto o POP previamente existente na organização quanto o POP-C auxiliam na promoção de um ambiente de trabalho gratificante.

Além de ser benéfico para quem executa a atividade, o POP-C também gera benefícios para quem treina profissionais, uma vez que facilita o planejamento e a execução do treinamento. Do ponto de vista do planejamento de ensino, segundo o treinador, o POP-C "ajudou a pensar nas informações mais importantes e se não o tivesse em mãos poderia esquecer de repassar alguma informação". Em relação à execução do ensino, também de acor- do com o treinador, o POP-C "com certeza [otimizou o tempo]. Se não tivesse com o padrão eu estaria até hoje explicando para ela", e promoveu a realização da atividade por P6 com o mínimo de intervenção do treinador: "só fez [a atividade] sozinho por causa do padrão [...]”. A importância do POP no contexto de treinamento também é explicitada por Mattsson, Salunke, Fast-Berglund, Li e Skoogh (2018). Os autores afirmam que a existência de instruções de trabalho é uma das condições para facilitar o ensino de comportamentos profissionais, de modo a garantir que os indivíduos, após realizarem a atividade descrita, obtenham um resultado de acordo com a necessidade do cliente.

Além de benefícios, o POP-C apresentou, na percepção de seus usuários, uma limitação: a extensão, percebida como excessiva pela quantidade de páginas ou de itens. P7 relatou que o tamanho do POP-C demanda tempo para a leitura e sugere sua diminuição. Já P6, embora não tenha se referido sobre a extensão do POP-C, afirmou sentir-se confusa em ter de olhá-lo (estava impresso) e executá-lo no computador. O treinador considerou que a extensão do padrão talvez tenha assustado os participantes. Sales et al. (2018), por meio de questionários respondidos por 247 trabalhadores da saúde, identificaram indicações de dificuldades de seguir os padrões por falta de tempo. Embora os autores não façam menção à extensão do POP, é possível supor que quanto maior sua extensão, maior será o tempo de leitura, o que diminui a probabilidade de realização da consulta ao padrão. Nesse sentido, a extensão do POP-C pode sinalizar a necessidade de alto investimento de tempo para ser lido, o que pode dificultar aos profissionais iniciarem a leitura ou se manterem lendo e realizando o que está escrito.

Contudo, a extensão do POP-C foi relatada como necessária, conforme fala do treinador: "[...] para uma pessoa inexperiente, é necessário que o padrão seja preciso”. Tais considerações levam a um questionamento: qual repertório de entrada deve ser considerado para a elaboração de um POP-C? Talvez seja mais prudente descrever um POP-C considerando um repertório básico, mesmo que extenso, e garantir o acesso a uma descrição completa e inequívoca da atividade, do que supor um repertório inicial aos trabalhadores, que podem deixar de apresentar tarefas necessárias à execução das atividades e dificultar, assim, a eficácia que é possibilitada pela realização do que está escrito no POP-C. Dessa forma, para diminuir uma possível objeção em seguir o extenso POP-C por parte dos profissionais, é importante que sejam realizadas intervenções a fim de destacar a relevância de seu uso na organização, tal como sugeriu o próprio treinador, quando afirma a necessidade de explicitar melhor o porquê de o padrão ser tão específico e extenso.

\section{Estudos 1 e 2: Discussão Integrativa}

O POP-C viabilizou, em 100\% das vezes, que profissionais com diferentes repertórios realizassem as atividades com uso do software de gestão, o que não ocorreu quando usaram os POPs previamente existentes na organização. Além disso, para profissionais que nunca haviam realizado a atividade descrita, seu uso gerou sentimentos como segurança e autonomia e, para o treinador desses profissionais, auxiliou na elaboração do treinamento. Como limitação, seu tamanho foi considerado extenso, mas também necessário a profissionais com repertório específico, uma vez que sua extensão é decorrente do grau de visibilidade das tarefas constituintes da atividade e da precisão e completude da descrição da atividade. Portanto, o uso de POP-Cs foi eficaz e beneficiou os profissionais da organização, ainda que tenha havido certas limitações.

Apesar dos significativos resultados relacionados à eficácia e aos benefícios dos POP-Cs no âmbito dos Estudos 1 e 2, eles precisam ser observados com prudência. Ainda há uma extensa lista de experimentações a serem feitas até que se possa chegar à conclusão 
de que a sentença comportamental, proposta aqui para o uso de profissionais na interação com softwares de gestão, seja confirmada como de entendimento inequívoco por diferentes profissionais e elaboradores de procedimentos de trabalho. Por exemplo, parece importante testar o POP-C somente com grupos de profissionais acima de 60 anos. Estudos com profissionais com mais idade são importantes, pois há uma tendência de que a taxa de profissionais com idade avançada aumente nas próximas décadas. Em outro extremo, também parece relevante testar o POP-C somente com participantes com idades entre 14 e 16 anos, por decorrência da baixa experiência profissional. A experimentação do POP-C (a) com mais participantes, (b) com atividades mais complexas, (c) com grupo controle, (d) com outros softwares exclusive os de gestão e (e) em hardwares diversos, como coletores de dados, smartphones e impressoras, entre outros, também são relevantes para a validação dos POP-Cs. Enfim, o que ocorreria com a eficácia e quais seriam os benefícios e as limitações do uso de POP-Cs nessas situações?

Também é relevante destacar que a utilização dos POP-Cs descritos nos estudos só foi uma realidade na organização estudada, pois teve o apoio da direção ao projeto. Problemas com a qualidade na prestação de serviços, acidentes de trabalho e necessidade de delegação das atividades motivaram a direção a promover o aperfeiçoamento dos POPs existentes. Considerando essa característica, quais seriam os resultados se os POP-Cs fossem testados em organizações nas quais o uso de procedimentos de trabalho é pouco valorizado?

Os resultados produzidos nesta pesquisa revelam que a noção de comportamento é orientadora aos profissionais que atuam com elaboração de procedimentos escritos. Tal contribuição aperfeiçoa o conhecimento produzido sobre a redação de POPs em áreas como gestão de processos, bem como evidencia a importância do psicólogo nas organizações como elaborador de procedimentos operacionais padrão, uma vez que o profissional é o especialista para lidar com o fenômeno a ser observado: o comportamento.

\section{Referências}

Associação Brasileira de Normas Técnicas (2005). ABNT NBR ISO 9000:2005 Sistemas de gestão da qualidade - Fundamentos e vocabulário. Rio de Janeiro: Associação Brasileira de Normas Técnicas.

Araújo, G. M. de. (2010). Elementos do Sistema de Gestão de SMSQRS: Sistema de Gestão Integrada (2 ed., Vol. 02). Brasil: Gerenciamento Verde Consultoria Editora.

Association of Business Process Management Professionals. (2013). BPM CBOK V3.0: Guia para Gerenciamento de Processos de Negócio Corpo Comum de Conhecimento. Brasil: ABPMP.

Botomé, S. P. (2013). O conceito de comportamento operante como problema. Revista Brasileira de Análise do Comportamento, 9(1), 19-46. Recuperado de https://periodicos.ufpa.br/index.php/rebac/article/view/2130/2433

Campos, V. F. (2014). Qualidade total: padronização de empresas (2 ${ }^{\mathrm{a}}$ ed.). Nova Lima: FALCONI Editora.

Empresa Brasileira de Serviços Hospitalares. (2014). Manual de Padronizą̧ão. Brasília: EBSERH. Recuperado de https://goo.gl/Xqm4py

Ferreira, A.B.H. (2004). Novo dicionário eletrônico da língua portuguesa Aurélio. Versão 5.11a Brasil: Positivo Informática.

Folha de São Paulo (2012, Outubro 21). Estagiária que injetou café na veia de idosa diz. que 'qualquer um se confunde'. Folha de São Paulo. Recuperado de https://goo. gl/sxyyiy

Gawande, A. (2009). The checklist manifesto: how to get things right. New York: Picador.

Gong, L., Li, D., Mattsson, S., Åkerman, M., \& Berglund, Å. F. (2017). The Comparison Study of Different Operator Support Tools for Assembly Task in the Era of Global Production. Procedia Manufacturing, 11, 1271-1278. https://doi.org/10.1016/j.promfg.2017.07.254

Harris, T. E. (2014). Applied Organizational Communication: Principles and Pragmatics for Future Practice ( $2^{\mathrm{a}}$ ed.). New York: Psychology Press.

Maranhão, M., \& Macieira, M. E. B. (2010). O processo nosso de cada dia: modelagem de processos de trabalho ( $2^{\mathrm{a}}$ ed.). Rio de Janeiro: Qualitymark.

Li, D., Mattsson, S., Salunkhe, O., Fast-Berglund, Å., Skoogh, A., \& Broberg, J. (2018). Effects of Information Content in Work Instructions for Operator Performance. Procedia Manufacturing, 25, 628-635. https://doi.org/10.1016/i. promfg.2018.06.092
Mattsson, S., Salunke, O., Fast-Berglund, Å., Li, D., \& Skoogh, A. (2018). Design concept towards a human-centered learning factory. Procedia Manufacturing, 25, 526-534. https://doi.org/10.1016/i.promfg.2018.06.121

Menn, J. P., Ulbrich, C., \& Seliger, G. (2018). First proof of concept for language independent learnstruments in special machinery assembly. Procedia Manufacturing, 21, 454-461. https://doi.org/10.1016/i.promfg.2018.02.144

Peixoto, A. L. A., Marques, P. E. D., Silva, R. A., \& Ferraz, V. A. (2015). Manual de elaboração de procedimentos operacionais e instruções de trabalho da Universidade Federal da Bahia. Salvador: EDUFBA. Recuperado de https://goo.gl/5uPLzH

Pereira, L. R., Carvalho, M. F., Santos, J. S., Machado, G. A. B., Maia, M. A. C., \& Andrade, R. D. (2017). Avaliação de procedimentos operacionais padrão implantados em um serviço de saúde. Arquivos de Ciências da Saúde, 24(4), 4751. https://doi.org/10.17696/2318-3691.24.4.2017.840

Rateke, E. C. M. (2014). Elaboração de manuais, procedimentos operacionais padrão e instruções de trabalho. Hospital Universitário Prof. Polydoro Ernani de São Thiago da Universidade Federal de Santa Catarina. Recuperado de http:// www.hu.ufsc.br/pops/pop-externo/download?id =71

Reeuwijk, L. P. van., \& Houba, V. J. G. (1998). Guidelines for Quality Management in Soil and Plant Laboratories (FAO Soils Bulletin - 74). Recuperado de http:// www.fao.org/docrep/W7295E/W7295E00.htm

Sales, C. B., Bernardes, A., Gabriel, C.S., Brito, M.F. P., Moura, A. A., \& Zanetti, A. C. B. (2018). Protocolos Operacionais Padrão na prática profissional da enfermagem: utilização, fragilidades e potencialidades. Revista Brasileira de Enfermagem, 71(1), 126-134. https://doi.org/10.1590/0034-7167-2016-0621

Silva, W. L. V. da, Duarte, F. M., \& Oliveira, J. N. de. (2004). Padronização: um fator importante para a engenharia de métodos.Qualit@s, 3(1), 1-15. Recuperado de http://revista.uepb.edu.br/index.php/qualitas/article/viewFile/35/27

United States Environmental Protection Agency [US EPA]. (2001). Guidance for Preparing Standard Operating Procedures (SOPs). Recuperado de https://www. epa.gov/sites/production/files/2015-06/documents/g6-final.pdf

Veja (2013). Campinas: falha bumana causou a morte de três pacientes após ressonância magnética. Recuperado de https://goo.gl/ZPtYN3 